\title{
The effects of auditory and visual interference on the immediate recall of melody
}

\author{
WILLIAM R. BALCH \\ Pennsylvania State University, Altoona, Pennsylvania
}

\begin{abstract}
College-age subjects recalled melodies or spoken sentences immediately after hearing them, by either pointing on a chart designed to interfere with a visual memory code or saying the equivalent responses, which should interfere with an auditory code. Pointing took significantly longer than saying for melodies, but not for sentences. A second experiment showed that this task $x$ materials interaction could be due to interference with contour per se rather than with visual coding of melody, because a similar effect was found for spoken contours consisting simply of the words "up" or "down." In a third experiment, pointing and saying tasks were modified both to control and to manipulate the amount of contour interference. Visual control stimuli for melodies were introduced, employing a marker that moved up or down. This time the task $\times$ materials interaction could be explained in terms of an auditory memory code for melody, but there were some problems with this interpretation. Interference related to contour, rather than to a specific modality, appeared to account best for the results of all three experiments.
\end{abstract}

In a well-known study, Brooks (1968) required subjects to recall a spoken sentence by saying a series of "yes" or "no" responses designed to interfere with an auditoryverbal memory representation of that sentence. This recall task took longer for subjects to complete than a pointing task in which they indicated the correct "yes" or "no" responses on a visual chart, on which the letters $Y$ and $\mathrm{N}$ were spatially arranged. Interestingly, the saying task became faster than the pointing one when the stimulus to be recalled was a visual pattern. Apparently, the pointing task interfered in this case with a visual-spatial memory representation of the presented pattern. Brooks's experiment was an early and effective demonstration of modality-specific immediate recall.

A recent interference experiment by Ohlsson and Ronnberg (1981) reinforced this notion of modality-specific short-term memory. Although the logic behind their experiment was similar to that of Brooks (1968), their method differed in two key respects. First, the same materials (lists of words) were presented either auditorily or visually. Second, interference was not manipulated within recall task itself. Rather, an interpolated 15 -sec interval was introduced, during which distracting nonsense syllables were presented via either modality. Just before recalling the original word list, subjects were required to

The research reported in this paper was supported by a Faculty Development Grant from Pennsylvania State University. The author would like to acknowledge the assistance of David Carothers, Kathy Crocetti, Beth Krisco, Dennis Muscatelli, Norma Ritz, and Joann Treese, and of Warren Witzig and Anthony Baratta. In addition, Steven Keele, Henry Roediger, and Andrea Halpern provided helpful comments on several methodological and theoretical points. Correspondence concerning the article or requests for reprints should be sent to William $R$. Balch, Department of Psychology, Pennsylvania State University, Altoona, PA 16603. -Steven W. Keele served as Action Editor on this manuscript. indicate the number of times certain letters had occurred in the nonsense material. Thus, interference was produced by a secondary task involving distracting experimental input. Despite these departures from Brooks's methods, the results were largely comparable. Compared with control conditions employing 15-sec delays without interfering material, both auditory and visual interference reduced recall accuracy of items originally presented in the same mode.

If verbal material heard by subjects is entered into memory via an auditory code, it might be expected that musical stimuli would also be stored in such a code. The little available evidence on modality-specific memory of music supports this hypothesis to some extent, but there are some unresolved questions. In one study, Massaro (1970) showed that simple pitch recognition can be impaired by the presence of an interfering tone interpolated between the target and comparison stimuli. Deutsch (1970), in another pitch-recognition study, used a six-tone interference pattern, which produced about a $32 \%$ error rate in recognition. However, interpolating spoken numbers led to only a $2 \%-6 \%$ eror rate. Although this result suggests a memory code for pitch that is highly specific to tones, it is surprising that so little interference occurred with the spoken numbers.

Verbal spoken and musical stimulation both involve auditory input, and presumably share at least some perceptual and memory processing mechanisms. Therefore, a modality-specific hypothesis should predict some degree of interference with musical memory by verbal stimulation. Although such interference was not found by Deutsch (1970), it might be induced if the stimulus to be remembered were more complex, that is, if the memory load were greater. Williams (1982) reported an experiment in which music students were tested for recognition of a par- 
ticular pitch in a seven-note melody after a silent interval of $10 \mathrm{sec}$, a 10 -sec period of auditory interference consisting of 20 random interpolated tones, or a $10-\mathrm{sec}$ silent interval followed by the random tones. Recognition was then tested by signaling a numerical position of the note to be recognized within the original melody, presenting a comparison tone, and having subjects indicate whether or not this test tone was the correct pitch. Performance in conditions in which auditory interference was present dropped to chance level or worse. As in the Deutsch (1970) and Massaro (1970) studies, tonal interference was very effective in reducing pitch recognition. Because Williams did not include any nonmusical form of interpolated auditory stimulation, whether his effect stemmed from interference specific to musical tones or to the auditory modality per se was not clear. Thus, one area of uncertainty in the literature, to be addressed in the present study, is whether auditory interference with the memory of a musical stimulus can be produced by a nonmusical but auditory means, such as a verbal saying task.

A second problem that needs to be investigated concerns the possibility that melodic memory coding may be only initially auditory. For instance, under some circumstances, a listener attempting to remember a melody might transform it into another code. In the present experiments, visual coding of melody will also be considered as a hypothesis.

There is some justification in the literature for supposing that auditory input can be transformed into a visual short-term memory code. One reason for such a view is that coding transformations appear to occur in either direction, on the basis of evidence from verbal studies. For instance, some visually presented stimuli are likely to be storable in an auditory memory code. In the study already cited, Ohlsson and Ronnberg (1981) found that auditory distractor material interfered with the recall of visually as well as auditorily presented words. This apparent visual-to-auditory-code transformation has also been demonstrated in another manner by Conrad (1964). When subjects recalled visually presented letters, erroneously recalled items shared auditory features with the correct ones. However, there is some evidence that recall errors also have an above-chance visual similarity to target items, even when those items are heard rather than seen (Laughery, Welte, \& Spector, 1973). This study showed that at least to some extent, auditorily presented letters can be represented visually in memory.

Williams (1982) reported a case in which melodies heard by subjects might also be stored visually in memory. In one of his experiments, musically experienced listeners were required to remember melodies by recognizing $10 \mathrm{sec}$ later which of three visually presented diagrams matched the contour of the original pattern. When melody contour recognition was thus visually tested, random tones interpolated before the recognition test did not affect subjects' accuracy compared with a control test without the interference. Remember that Williams found this audi- tory interference lowered accuracy of an auditory recognition task to chance level. Taken together, these results suggest that different memory coding mechanisms might be engaged depending on the memory task employed.

A third difficulty emerges in studying memory of melody. Confounded with the question of how melodies are stored in short-term memory is that of what information carried by the melody is best remembered. In particular, the up-down contour of a melody is a type of information that predominates in the short-term recognition of a novel musical stimulus (Dowling, 1978; Dowling \& Bartlett, 1981). These studies have shown that a test stimulus, having intervals and pitches different from those of a target item, will still tend to be mistaken for the original if the contour has not been altered. However, listeners can easily recognize as new those melodies whose contours are different (Bartlett \& Dowling, 1980).

Although important in melody recognition, contour is not unique to musical materials. For instance, consider the melody notated in Figure 1A. The contour of this melody is expressed visually by the notation, and may also be expressed by playing the melody. Still another way of conveying the contour is verbal. For example, the spoken words "up, up, down, up, up, down" describe the same contour information. That is, the second note goes up from the first, the third goes up from the second, the fourth goes down from the third, and so forth. This last representation will be called a spoken contour, and is important to the design of this study.

In Experiment 1, Brooks's (1968) methods were adapted to test for visual memory coding of melodies. Similar yes-no pointing and saying tasks were used. In Brooks's procedure, recall of sentences was contrasted with that of visual figures. The main departure in the present experiment is that seven-note melodies, like that shown in Figure 1A, were substituted for the visual designs. That is, either melodies or sentences were employed as materials. As in Brooks's study, evidence for visual coding would occur if an appropriate task $\times$ materials interaction were found. In other words, the visually interfering pointing task should take considerably longer than the saying task with the melodies. However, for the sentence material this difference should either not occur or should even be reversed: The auditorily and verbally interfering saying task should take longer than pointing.

\section{EXPERIMENT 1}

\section{Method}

Subjects. Thirty-two undergraduate volunteers (14 females and 18 males) participated in this experiment. All were enrolled in the introductory psychology course at Pennsylvania State University, Altoona; each received a small amount of academic credit for participating. Their median number of years of lessons on a musical instrument was 2.0.

Materials and Design. One group of 16 subjects ( 7 males and 9 females) was tested on recall of seven-note melodies. Every melody was a random tonal sequence, consisting of intervals of either one or two diatonic steps up or down. (Each successive white key on 
a piano keyboard is an example of a diatonic step.) Interval probabilities were as follows: $p(+1)=1 / 3, p(-1)=1 / 3, p(+2)=1 / 6$, and $p(-2)=1 / 6$. Eight experimental sequences were presented on high-fidelity tape-recording equipment: (1) $C_{5} E_{5} F_{5} E_{5} D_{5} C_{5} B_{4}$; (2) $D_{5} E_{5} G_{5} A_{5} G_{5} F_{5} G_{5}$; (3) $E_{5} G_{5} E_{5} D_{5} E_{5} D_{5} C_{5} ;$ (4) $F_{5} D_{5} B_{4} C_{5}$ $D_{5} C_{3} D_{5} ;(5) G_{5} E_{5} F_{5} D_{5} B_{4} C_{5} D_{5} ;(6) E_{5} D_{5} B_{4} C_{3} B_{4} A_{4} B_{4}$; (7) $F_{5} G_{5} E_{5} C_{5} D_{5} B_{4} C_{5}$; and (8) $G_{5} F_{5} E_{5} F_{5} A_{5} B_{5} G_{5} .{ }^{.}$The melodies were originally played by a trained musician on an alto Yamaha recorder. The tempo was controlled at two notes per second by a Franz flash-beat metronome.

Another group of 16 subjects had the same median level of musical experience as did the first one and also the same proportion of males and females. This second group was tested on the recall of sentences. The eight sentences were: (1) "Bring the box to the customer." (2) "In the room a man painted." (3) "An orange sun lit the sky." (4) "A dog and a cat fought." (5) "Peaches were a dollar a pound." (6) "Can the bather stand the heat?" (7) "Make a pie an hour, please." And (8) "The attendant came in a minute." Each sentence contained six words so that there could be six "yes""no" responses, just as in the case of the seven-note melodies. This verbal material was tape-recorded by a male voice speaking at the rate of two words per second.

Together then, the melodies group and the sentences group defined the materials variable. The other main independent variable was recall task. Each of two tasks was performed by every subject in the experiment, creating a $2 \times 2$ mixed factorial design.

In the saying task, the subjects recalled each melody or sentence by speaking a series of six "yes" or 'no' responses. For melodies, the experimenter gave the one-word instruction "up" or "down" immediately after a melody was presented. If the instruction was "up," the subjects were to say "yes" each time a note went up in pitch from the last one; the subjects were to say "no" each time it did not. For the melody illustrated in Figure 1A, the response to the "up" instruction was to be: "yes yes no yes yes no." However, if the "down" instruction was given, the subject was to say "yes" every time a note went down and "no" every time it did not. Thus, if the "down" instruction was given just after the melody in the figure was presented, the response was to be "no no yes no no yes." The two different instructions ("up" or "down") were introduced to discourage subjects from encoding the stimulus into "yeses" and "nos" during its presentation. Since the subjects did not know the instruction until after the stimulus was heard, their recall times reflected the amount of interference of the task with memory coding of the stimulus.

\begin{tabular}{|c|c|c|}
\hline 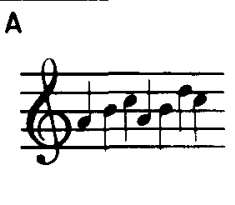 & 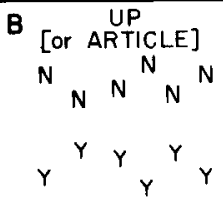 & 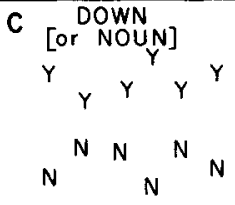 \\
\hline$---ー-ー$ & $E$ & 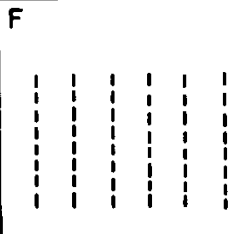 \\
\hline
\end{tabular}

Figure 1. Plate A illustrates a typical melody heard by the subjects in Experiment 1. Plates $B$ and $C$ show the visual charts used for the pointing task in Experiments 1 and 2. Plate D shows the control chart used for the saying task. The computer-generated visual contours in Experiment 3 are depicted in Plate E, but in the displays themselves, one horizontal bar successively occupied each of the six indicated positions in a left-to-right direction. Plate $F$ shows the chart used in Experiment 3 for the pointing task.
For the sentences, the saying task again required subjects to give a series of six spoken "yes" or "no" responses. The only difference was the instruction given by the experimenter just after the stimulus was presented. Either "noun" or "article" was the instruction used. In the former case, the subjects said "yes" each time a word in the sentence was a noun and "no" each time it was not. In the latter case, a "yes" response was required whenever a word was an article ("a," "an," or "the") and "no" whenever it was not. For instance, suppose the sentence was "A tree was blocking the view." After the "noun" instruction, the recall would be: "no yes no no no yes." After the "article" instruction, it would be: "yes no no no yes no."

The other recall task was pointing. According to this task, the subjects indicated their "yes" or "no" respnses by pointing to successive Ys and Ns on a visual chart, in a left-to-right direction. For example, the chart in Figure 1B was used for pointing responses to the "up" instruction (for the melody group) or to the "article" instruction (for the sentence group). The chart in Figure $1 \mathrm{C}$ was used for recall under "down" or "noun" instructions. The two charts were designed to create maximal visual interference with melodic contour when the subjects pointed their recalled responses.

Two additional methodological variables were employed as counterbalances. First, half of all subjects performed the saying task on the second, fourth, seventh, and eighth melody (or sentence) in the experimental set; the pointing task was performed on the other items. For the other half of the subjects, this assignment was reversed. The subjects performed the saying tasks on Items 1, 3, 5 , and 6 and the pointing tasks on the other items.

A second counterbalanced variable concerned the assignment of instructions to items. Half the subjects received "up" (or "article") instructions after Items 1, 4, 5, and 7 and "down" (or "noun") instructions after the other items. The remaining subjects heard "down" (or "noun") instructions with Items 2, 3, 6, and 8 and "up" (or "article") instructions with the other items. Thus, a total of four combinations of these counterbalanced conditions were assigned to the different subjects in each of the two groups (melodies or sentences).

Procedure. In individual sessions, the subjects were familiarized with tasks to be performed through the use of five warm-up items. They were then tested on the immediate recall of eight experimental stimuli. The procedure for these experimental trials will be described first.

On each trial, the subjects were told in advance whether the task would be pointing or saying. If the pointing task was to be performed, the two charts illustrated in Figures $1 B$ and $1 C$ were set on a stand at arm's length in front of the subjects. The former chart was labeled "Up" (for the melody group) or "Article" (for the sentence group) and was used for those instructions. It was always placed on the left. The other chart was labeled "Down" (with melodies) or "Noun" (with sentences) and was used after this instruction. On these pointing trials, the subjects were instructed to hold a wooden pointer ready in their hands, for indicating their answers on one of the charts.

For the saying task, no pointer was required. In order to make the saying task as comparable as possible to the pointing task, two charts were set in front of the subjects for each trial. These charts consisted simply of six blanks. (See Figure 1D.) One was labeled "Up" (for the melody group) or "Article" (for the sentence group). Again it was placed on the left. The other was labeled "Down" (for melodies) or "Noun" (for sentences). The subjects were told to look at whichever chart was indicated by the instructions and to use the blanks to keep track of the number of responses in their answers. This feature of the saying task was deemed necessary because the pointing charts offered this advantage to the subjects.

After informing the subjects whether a particular trial would require saying or pointing, the experimenter started the tape recorder. First, a male voice spoke the item number, and then, after $2 \mathrm{sec}$, the melody (or sentence) was heard. Immediately afterwards, the experimenter stopped the tape recorder with a silent "pause" switch 
and spoke a one-word instruction. This instruction was either "up' or "down" for the melody group, or "noun" or "article" for the sentence group. The subjects then performed the required recall task (as explained in the Materials and Design section). The time required for recall was recorded on each of the eight experimental trials. If a subject reported "losing" an item during the recall task, this stimulus was repeated at the end of the series. The subjects were instructed not to repeat items simply if they thought their recall was not perfect, but to do so only if they had forgotten the item in its entirety. Only about $3 \%$ of the items needed to be repeated.

To acquaint the subjects with the task requirements, five warmup trials were given at the start of the session. Warm-up stimuli were of the same type used in the experimental series. After the first warm-up item had been presented, the experimenter explained how an item would be recalled according to the pointing or saying task and how the "yes" or "no" answers would depend on the instruction given immediately after the presentation of the stimulus. Thus, the melody subjects were shown how to use the "up" or "down" instruction, and the sentence subjects were told how to use the "noun" or "article" instruction. The first warm-up example was repeated as often as necessary for the subjects to understand how the tasks worked. Then four more trials were given, during which the subjects were required to recall a new item under each of the four task combinations possible for any single subject. That is, each participant did the pointing and the saying task with each of the two instructions used for his or her group. These last four warm-up trials were conducted just like the experimental ones, with the following exceptions. After having recalled each item, the subjects received feedback on the correctness of the answer and then heard the item repeated once while the right answer was indicated. They also were allowed to ask questions. These procedures helped ensure that the subjects understood the task requirements.

\section{Results and Discussion}

The results of this experiment are summarized in Table 1 , which shows the mean recall times and standard deviations for the four combinations of materials and tasks.

Neither task nor materials, as main effects, significantly influenced recall times. The mean time for completing the pointing task was $10.23 \mathrm{sec}$, and the saying task required a mean of $9.66 \mathrm{sec}[\mathrm{F}(1,30)=2.39, \mathrm{p}>.10]$. Melodic materials averaged $10.53 \mathrm{sec}$, as opposed to $9.35 \mathrm{sec}$ for sentences $[\mathrm{F}(1,30)=.94, \mathrm{p}>.10]$.

The result of major interest is the significance of the task $\times$ materials interaction $[F(1,30)=14.12$, $p<.001]$. As Table 1 shows, recalling melodies by pointing required $11.51 \mathrm{sec}$, about $2 \mathrm{sec}$ longer than it took to remember the melodies with the saying task $(9.54 \mathrm{sec})$. This difference is significant $[t(15)=3.74$, $\mathrm{p}<.002]$. However, the pointing task did not interfere more with sentence recall. As a matter of fact, the direc-

Table 1

Mean Recall Times and Standard Deviations (SDs) in Experiment 1 as a Function of Task and Materials

Task

\begin{tabular}{lrrrrr}
\cline { 6 - 6 } & \multicolumn{2}{c}{ Pointing } & & \multicolumn{2}{c}{ Saying } \\
\cline { 2 - 3 } \cline { 6 - 7 } Materials & Mean & SD & & Mean & SD \\
\hline Sen- & & & & 9.76 & \\
tences & 8.95 & 2.57 & & 9.53 \\
Melodies & 11.51 & 4.80 & & 9.54 & 4.37 \\
\hline
\end{tabular}

Note-All entries are expressed in seconds. tion of the difference was reversed, although it was not significant. The pointing method of sentence recall required $8.95 \mathrm{sec}$, about $1 \mathrm{sec}$ less than it took to remember by saying $(9.76 \mathrm{sec})[\mathrm{t}(15)=1.55, \mathrm{p}>.10]$. Together, these results indicate that pointing definitely interfered more than saying with melody recall, but not with sentence recall. This task $\times$ materials interaction is essentially the same one found by Brooks (1968), except that, in Brooks's study visually presented figures-not auditorily presented melodies-were compared with sentences. Thus, the recall of melody, like that of visual figures, appears to be subject to interference from a concurrent visual task that is incongruous with melody contour.

\section{EXPERIMENT 2}

An important problem in interpreting the visual interference effect found in Experiment 1 is that the sentence materials, used for comparison purposes, contained no up-down contour information. Therefore, whether the pointing task interfered with contour per se or with a visual code specific to the presentation of melody is not clear. In Experiment 2, spoken contours were introduced as stimuli. These items expressed the same contours as the melodies in Experiment 1, but were spoken series consisting of the words "up" and "down." For instance, the first melody in that experiment was: $C_{5} E_{5} F_{5} E_{5} D_{5}$ $C_{5} B_{4}$. The first item in Experiment 2 was thus: "up up down down down down." The task, whether pointing or saying, was simply to indicate "yes" if a given word in the sequence were "up" (or, on half of the trials, "down") and "no" if it were not.

If pointing interferes only with contour memory, it should slow the recall of the spoken contours as much as that of the melodies. However, suppose the visual interference obtained in Experiment 1 was to some extent specific to the presentation of melodic as opposed to verbal materials. Then there should be less interference of pointing, relative to saying, with the recall of spoken contours. Indeed, the effect should even be reversed.

\section{Method}

Subjects. Sixteen undergraduates (seven females and nine males), enrolled in introductory psychology at the Altoona Campus of Pennsylvania State University, participated in this study. Their median number of years of lessons on a musical instrument was about 2 . Thus, these subjects were comparable to those in the two groups of Experiment 1 .

Materials, Design, and Procedure. Methodologically, this experiment was conducted in precisely the same way that Experiment 1 had been conducted with the melody group. Again, the subjects performed "yes"'-"no" pointing and saying tasks; the charts were the same as before. This time, the subjects heard spoken contours: series of six words ("up" or "down"). Each item matched the contour of its counterpart melody. In other words, whenever the pitch had gone up in a melody used in Experiment 1, the word "up" was spoken in the verbal item. Whenever the pitch had gone down, the word "down" was used. In either task, when the "up" instruction was given after the item, the subjects indicated "yes" when the word was "up" and "no" when it was not, and likewise for "down." Thus, the output of the subjects' recall was just the same: 
six "yes" or "no" responses. The counterbalancing scheme, according to which a specific task and instruction were assigned to a particular item, was identical to that of Experiment 1.

\section{Results and Discussion}

The results for spoken contours paralleled those obtained with melodies in Experiment 1. When the subjects pointed their recall of the verbal "ups" and "downs" on the visually interfering charts, they required an average of $7.79 \mathrm{sec}$ for each spoken contour $(\mathrm{SD}=2.08 \mathrm{sec})$. However, they took only $5.97 \mathrm{sec}(\mathrm{SD}=1.65 \mathrm{sec})$ to say "yes".-"no" responses to the same contours. The pointing minus saying difference was thus $1.82 \mathrm{sec}$, which was significant $[t(15)=5.57, \mathrm{p}<.001]$. It is very similar to the 1.95-sec difference obtained with melodies used in Experiment 1. The comparison between the two differences is nonsignificant $[\mathrm{t}(30)=.20, \mathrm{p}>.10]$.

Considered together, Experiments 1 and 2 indicate that the recall of up-down contour is disrupted more by a visual pointing task than by an auditory saying one. However, contour need not be expressed via melodies only. The pointing task interferes just as effectively with memory of the spoken contours, when the same information is conveyed semantically rather than musically. On the other hand, sentence recall is not subject to the same interference. Unlike the melodies and spoken contours, sentences provided no up-down information. Apparently, contour per se is what the pointing task interfered with.

\section{EXPERIMENT 3}

Quite possibly the contour-specific interference effect obtained in the first two experiments overpowered any modality-specific interference of the saying task with auditory memory coding. In Experiment 3, contour was controlled simultaneously in two respects. First, the pointing and saying tasks were modified to be equivalent in the amount of contour interference that they provided. In one version of each task, recall responses were congruent with the contour of the stimulus item. In the other, responses were incongruent with contour. Thus, the degree of contour interference engaged by the recall task was both factored out and independently manipulated.

A second control for contour was provided by contrasting recall of melodies with that of equivalent visual contours. To produce these latter stimuli, a computergenerated visual marker went up or down across a CRT screen at the same rate that the notes in the melodies were played.

In these ways, Experiment 3 provided a fair test for the hypothesis of auditory coding for melodies. With contour controlled, this hypothesis would predict that saying should be slower than pointing for the melodies. However, this effect should be less, or even reversed, for the visual contours. This prediction can be made because the saying task is designed always to interfere auditorily and specifically with the recall of melodies, whereas the pointing task provides no auditory interference. This difference in the tasks applies regardless of the level of contour in- terference (i.e., whether the task is congruent or incongruent with contour.

\section{Method}

Subjects. Seventy-two undergraduates currently taking introductory psychology at Pennsylvania State University, Altoona, participated in this experiment. None of the subjects had been in either of the previous experiments.

Design. Four different groups of 18 subjects each were tested according to one of four combinations of materials (melodies or visual contours) and recall task (pointing or saying). In individual sessions, every subject recalled items under two different instructions: either to make responses congruent with the contour of the stimulus or else to make them incongruent. This variable will be referred to as contour condition. Thus, this experiment was a $2 \times$ $2 \times 2$ mixed factorial design.

Material. Half the subjects recalled each of 12 experimental melodies, generated and recorded exactly as in Experiment 1 . The only difference was that there were just six notes in each, rather than seven. The set of melodies was: (1) $F_{5} E_{5} G_{5} E_{5} F_{5} D_{5}$; (2) $E_{5} D_{5}$ $C_{5} D_{5} F_{5} E_{5}$; (3) $D_{5} E_{5} F_{5} A_{5} G_{5} F_{5}$; (4) $G_{5} E_{5} F_{5} D_{5} B_{4} C_{5}$; (5) $F_{5}$ $\mathrm{G}_{5} \mathrm{E}_{5} \mathrm{C}_{5} \mathrm{D}_{3} \mathrm{~B}_{4} ;(6) \mathrm{C}_{5} \mathrm{D}_{5} \mathrm{~B}_{4} \mathrm{G}_{4} \mathrm{~A}_{4} \mathrm{C}_{5} ;$ (7) $\mathrm{E}_{5} \mathrm{C}_{5} \mathrm{~B}_{4} \mathrm{C}_{5} \mathrm{~B}_{4} \mathrm{C}_{5}$; (8) $C_{5} E_{5} D_{5} E_{5} G_{5} A_{5}$; (9) $E_{5} G_{5} E_{5} D_{5} E_{5} D_{5} ;(10) E_{5} D_{5} B_{4} C_{5}$ $B_{4} A_{4} ;$ (11) $G_{5} F_{5} E_{5} F_{5} A_{5} B_{5} ;(12) D_{5} E_{5} D_{5} C_{5} A_{4} F_{4}$. These materials were generated and recorded exactly as those in Experiment 1.

The other subjects recalled visual contours. These stimuli were programmed on an Apple II microcomputer. In Figure 1E, the nature of these visual items is illustrated. A horizontal marker successively occupies different positions on a series of six vertical bars. Figure 1E shows the visual contour corresponding to the fourth melody $\left(G_{5} E_{5} F_{5} D_{5} B_{4} C_{5}\right)$, which is: down up down down up. That is the second bar is lower than the first, the third is higher than the second, the fourth is lower than the third, and so forth. It is important to point out that the marker appears in only one position at a time and that it remains at each place for $.5 \mathrm{sec}$. Thus, the visual "tempo" is equivalent to that of the melodies (two beats per second). The up or down change in position was a constant distance, corresponding to a visual angle of about $1^{\circ} 30^{\prime}$. The height of the vertical bars was $12^{\circ} 15^{\prime}$, the width, $0^{\circ} 30^{\prime}$, and the distance between each, $2^{\circ} 30^{\prime}$. These stimuli were designed and piloted to present the same contour information as the melodies and to create, as nearly as possible, a visual analogue to the melodic items, while providing a clear and undistracting visual display.

Recall task. The pointing task was modified by having the subjects point to a chart consisting of only six dotted lines (see Figure 1F). With a wooden pointer, the subjects indicated either the same contour (for the congruous task) or the inversion of the contour (for the incongruous task). The subjects were instructed to point "somewhere near the middle" of the leftmost line to indicate the first note or visual position. The precise spot was unimportant. Then the subjects were to move the pointer up or down, in a left-to-right manner, to positions on the subsequent lines that correctly indicated either the contour or the inversion of the contour. The distance moved in each up or down change was unimportant. However, the subjects were told to make their position changes definite enough that it would be clear to the experimenter whether "up" or "down" was being indicated.

The saying task required some piloting. The words "up" and "down' were considered, but this task would require only five responses to the six-note melodies or visual contours, whereas the pointing task required six. Instead, it was decided to have the subjects say six numbers that expressed contour. Starting with "five" for the first note (or visual position), the subjects increased the number by one each time the note (or position) went up and decreased the number by one each time it went down. For instance, for the sixth melody $\left(C_{5} D_{5} B_{4} G_{4} A_{4} C_{5}\right)$, the subject would say: "five six five four five six" for congruent contour conditions or "five four five six five four" for incongruent contour conditions where 
the pattern was inverted. The same responses would be given for the sixth visual contour. Although the subjects were asked to increase or decrease numbers by one, they were told it would be permissible to accidentally change the number by more. The important point was to express the directions of changes in the stimulus. No chart was used with the saying task in this experiment. This decision to simplify the task was made primarily because, in the previous experiments, the subjects had reported that the blank charts (see Figure 1D) seemed unnecessary for performing the task.

Contour condition. Immediately after the melody or visual contour stimulus had been presented, the experimenter gave a one-word instruction to the subjects. For the congruous contour condition, the instruction was 'same.' In this case, the subjects pointed or spoke numbers in the same up or down directions that occurred in the melodic or visual items. Similar conditions had been used in melodic recall studies by Davies and Jennings (1977), who required subjects to draw contour diagrams, and by Hair (1977), who had her first-grade subjects give verbal descriptions of contour.

In the incongruous condition, the instruction given was "reverse." The subjects then recalled the melody or visual contour by inverting it, that is, by reversing it with respect to the up-down dimension. For instance, suppose the contour of the original item had been: up up down down up. After the "reverse" instruction, the correct answer would be: down down up up down, as indicated either by pointing or saying. Thus, this incongruous condition introduced contour interference.

The assignment of "same" or "reverse" instructions to specific stimulus items was counterbalanced. The result was that half the subjects performed the instructions in one random order, and the other half performed them in the opposite random order. In other words, they performed "reverse" on every item for which the other subjects had done "same," and vice versa.

Additional accuracy scoring. As in the previous experiments, the primary dependent measure was recall time. However, in this experiment, the subjects were also scored on response accuracy. There were two justifications for this precaution. First, it seemed advisable to check that the subject understood the "reverse" instruction and were using it, rather than reverting unknowingly to recalling the same contour as in the presented item. As it turned out, the data also allowed additional analyses that were of theoretical interest in interpreting the recall time data. These analyses will be discussed in connection with the results of this experiment.

Procedure. The procedure was essentially the same as that used in the first two experiments. As before, an item was presented, and then a one-word instruction ("same" or "reverse") was given. Immediately afterwards, the subjects indicated their recall according to the prescribed task. In addition to being timed by the experimenter, the subjects' responses to each stimulus were independently recorded. ${ }^{2}$

\section{Results and Discussion}

In Figure 2, the mean response times for the eight combinations of experimental conditions are shown. A threeway analysis of variance revealed that all three main effects were significant.

An unanticipated effect, which will be referred to later in the General Discussion section, is that of materials. Visual contours took an average of about $3 \mathrm{sec}$ less time to be recalled than did melodies with the same up-down information $[\mathrm{F}(1,68)=20.46, \mathrm{p}<.001]$. This advantage cannot be explained by the fact that one of the recall tasks-pointing-was very similar in form to the visually presented stimuli. The subjects in the saying groups alone still were faster in recalling the visual contours than in remembering the melodies $[\mathrm{F}(1,34)=11.32, \mathrm{p}<.01]$.

Not surprisingly, items were recalled more quickly un-
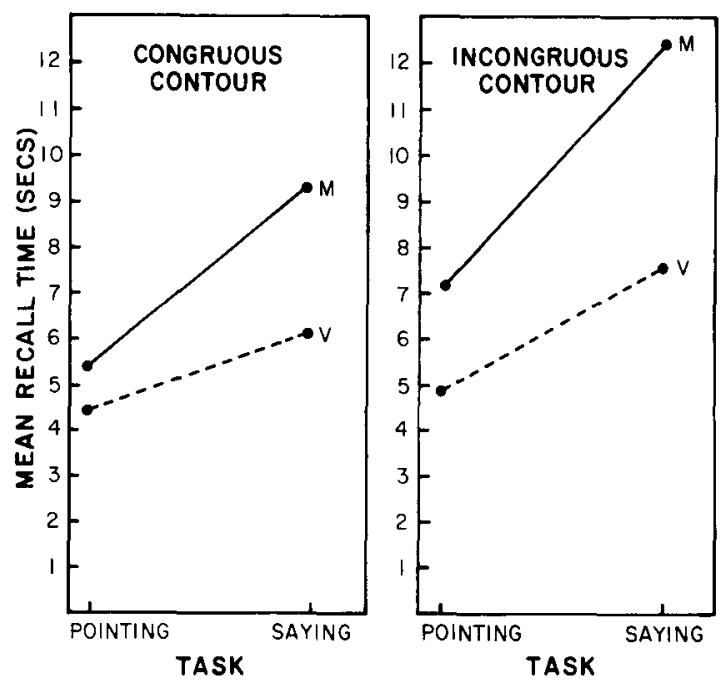

Figure 2. Mean recall times for the conditions of Experiment 3, where $M$ represents melody groups and $V$ represents visual contour groups.

der the congruous contour condition than under the incongruous one (by $1.65 \mathrm{sec}$ ) $[\mathrm{F}(1,68)=84.86$, $\mathrm{p}<.001]$. The latter condition, in which the subjects reversed up-down directions in their recall, introduced contour interference. The highly reliable effect of this manipulation in the present experiment is consistent with the disrupting effects of the visually interfering charts on the recall of stimulus materials expressing contour information, an effect found in Experiments 1 and 2.

Looking at the main effect of recall task, pointing was consistently faster than saying $[F(1,68)=30.45$, $\mathrm{p}<.001]$ by an average of about $3.5 \mathrm{sec}$. It seems highly likely that some of this effect was due to cognitive differences between the two tasks. Compared with the pointing task, the saying task required an additional conversion into numeric symbols.

The interaction between recall task and contour condition was significant $[F(1,68)=10.00, p<.01]$. The pointing task required $1.09 \mathrm{sec}$ longer to invert, whereas this difference was $2.21 \mathrm{sec}$ for the saying task. This interaction is not conclusive regarding modality-specific memory coding, because it could have stemmed largely from cognitive differences between the pointing and saying tasks.

A more interesting and unexpected interaction occurred between materials and contour. Compared with recalling congruous contours, reversing the contour required only about $1 \mathrm{sec}$ more for visual items. However, for melodies, this incongruous contour condition took about $2.3 \mathrm{sec}$ longer than did the congruous one. This result produced a significant interaction $[F(1,68)=12.57$, $\mathrm{p}<.001]$. This materials $\times$ contour interaction will be interpreted further in the General Discussion section.

The three-way interaction among the independent variables-materials $\times$ contour $\times$ task-was not significant $[F(1,68)=.03, p<.10]$.

The key result of theoretical interest in this experiment 
is the recall task $\times$ materials interaction, which tests for the auditory coding of melodies under conditions that were controlled for contour. This interaction was significant $[F(1,68)=4.58, p<.05]$. Although saying took longer than pointing for both types of materials, this difference was relatively small for the visual contours: $2.16 \mathrm{sec}$. However, it was much larger for the melodies, which required $4.89 \mathrm{sec}$ longer to be recalled by saying than by pointing. The particular difficulty of saying the responses to melodies is consistent with the conclusion that an auditory memory code for melody was being interfered with by a concurrent task engaging the same modality.

However, there are at least two alternatives to this interference with-an-auditory-code interpretation of the task $x$ materials interaction. One of these alternatives I shall call the differential trace-loss explanation. According to this interpretation, the memory traces of visual contours fade more quickly than those of auditory ones. (Indeed, when I tested myself on the visual condition, it seemed I had to rush recall occasionally to keep from forgetting the contour.) Since the saying task took longer for the subjects to complete, they might have had to hurry up their recall of visual contours on this task in order to prevent loss of the quickly fading visual trace. Therefore, there would not be much additional time taken for saying (as opposed to pointing) the visual contours. However, the presumably more durable auditory trace would have allowed the subjects to take as much time as they needed for saying the auditory contours. Accordingly, the saying minus pointing difference would be larger for the auditory contours. Note that this explanation does involve the assumption that there is a modality difference in memory coding between visual and auditory stores. Yet this difference is considered to be simply one of fading with time, and there is no assumption that the saying task interferes more with an auditory code for melodies.

A second alternative interpretation of the task $x$ materials interaction explains it in terms of interacting difficulty levels. Being divergent rather than crossing over, the interaction might be accounted for merely by assuming that there is superadditivity between the factors of materials and task. The saying task is more difficult than pointing, and auditory contours are more difficult to recall than visual ones. These two time-consuming conditions might interact to produce an extradifficult task when subjects are required to say the auditory contours. This explanation involves no assumptions of modality-specific coding at all.

These two alternative hypotheses can be at least partially tested by additional analyses of accuracy as well as the speed of recall.

Accuracy scoring results. A secondary analysis of variance was performed on the accuracy data, mainly to check how valid the interpretations of the response time data were. The mean accuracies for each of the eight conditions in Experiment 3 are shown in Table 2.

If the differential trace-loss hypothesis were true, the subjects should show a drop in accuracy under the condition of saying visual contours. This drop should occur be- cause the subjects would be hurrying to recall the more quickly fading visual contours.

There is partial support for the differential trace-loss hypothesis in terms of the interaction between task and materials (see Table 2). This interaction is significant $[F(1,68)=4.06, p<.05]$, indicating that the accuracy of the saying task is lower than that of the pointing task for visual contours. However, the direction of the effect is reversed for melodies.

As Table 2 shows, this interaction is mainly a reflection of the data for the incongruous condition (the bottom half of Table 2). The analysis of variance for the accuracy data also bears out this conclusion. Since the three-way interaction-task $\times$ materials $\times$ contour condition-is nearly significant $[F(1,68)=3.17$, $\mathrm{p}<.08$ ], separate interactions were calculated for congruous and incongruous contour conditions. The materials $\times$ recall task interaction was significant for the incongruous condition $[F(1,68)=6.00, p<.05]$ but not for the congruous condition $[\mathrm{F}(1,68)=.75, \mathrm{p}<.10]$.

This pattern of results indicates that, for the incongruous contour conditions only, there could have been some "artificial" speeding up of the saying responses to visual items, due to rapid fading of the memory trace for these stimuli. However, this problem does not apply to congruous-condition data. This congruous condition also was significantly less difficult (79.5\% accuracy) than was recalling the inverted incongruous contours $(72 \%$ accuracy) $[F(1,68)=20.44, p<.001]$.

Another potentially embarrassing aspect of the task $x$ materials interaction for accuracy data (in the incongruous condition) is that it is, in effect, a reversal of the corresponding latency-data interaction. It was this latter interaction, in which saying was more difficult for melodies and less difficult for visual contours, that was taken as evidence of auditory-specific melody coding. Again, however, the problem does not apply to the congruouscondition data for accuracy.

Perhaps the most serious problem with the auditory coding hypothesis is that slower saying times for melodies might be explained in terms of an interaction of difficulty levels, an argument discussed in the preceding section. If the task $\times$ materials interaction for response times reflected only superadditivity of two difficult conditions (i.e., saying and melodies), then the accuracy data should also reflect greater difficulty for both conditions. It is true that melodies were more difficult $(69 \%$ accuracy) than

Table 2

Mean Percent Accuracies in Experiment 3 as a Function of Recall Task, Materials, and Contour Condition

\begin{tabular}{lcc}
\hline & \multicolumn{2}{c}{ Task } \\
\cline { 2 - 3 } Materials & Pointing & Saying \\
\hline \multicolumn{2}{c}{ Congruous } & Condition \\
Visual Contours & 86.5 & 86.9 \\
Melodies & 69.6 & 75.0 \\
& Incongruous Condition & \\
Visual Contours & 85.3 & 75.4 \\
Melodies & 60.4 & 67.2 \\
\hline
\end{tabular}


were visual contours $(83 \%$ accuracy) $[F(1,68)=32.85$, $p<.001]$. However, the effect of recall task on accuracy was not significant $[\mathrm{F}(1,68)=.16, \mathrm{p}>.10]$. The mean accuracy for the pointing task was $75.2 \%$, whereas that for saying was $76.1 \%$. Therefore, the interaction-ofdifficulty-levels hypothesis is not itself a wholly satisfactory explanation of the task $\times$ materials interaction obtained with the response time data.

A post hoc analysis of response time data. The accuracy-data analysis revealed some problems of interpretation with the auditory coding hypothesis: the dropoff in accuracy for subjects saying visual contours, and the reverse interaction from that obtained with the latency data. Both problems were limited to the more difficult and time-consuming incongruous contour condition. Accordingly, a recalculation of the crucial materials $\times$ task interaction of response times-including only congruous contour data-should provide a less ambiguous test of the auditory coding hypothesis. This interaction, illustrated by the left half of Figure 2, was significant $[F(1,68)=$ $6.50, \mathrm{p}<.05]$. It reflects the fact that for melodies the saying minus pointing difference in recall times was greater than that for visual items under the easier congruous contour condition, in which visual trace loss or reversed accuracy data were not problems.

The largest remaining problem for the auditory coding hypothesis is the interaction-of-difficulty-levels explanation. Although it did not extend to the accuracy data, it is hard to rule out if one presumes that the superadditivity of difficulties is limited to response times. It can only be pointed out that this problem seems to be more applicable to the incongruous contour task, but to be less severe in the case of the congruous one (left side of Figure 2). In this latter condition, saying is only $1.59 \mathrm{sec}$ slower than pointing for the visual contours. However, it is $4.02 \mathrm{sec}$ slower than pointing for the melodies, which is about two and a half times the difference obtained for visual contours. Considering both this proportionately large increase in the saying minus pointing difference and the comparable accuracies of the saying and pointing tasks, a plausible, but certainly not conclusive, case can be made for an auditory interference effect with at least the congruous contours. It does not seem unreasonable, given Brooks's (1968) evidence for modality-specific coding of spoken sentences, to expect a comparable result for auditorily presented melodies. However, the more impressive and less problematic interference effect obtained was the main effect of contour condition, in which inverting contour clearly took longer and was less accurate than recalling congruous contours. In the following discussion, it will be argued that the results of all three experiments are best accounted for if interference with a more abstact contour memory coding is assumed.

\section{GENERAL DISCUSSION}

Experiments 1 and 2 showed the conditions under which a visual pointing task, adapted from Brooks (1968), in- terferes with the recall of auditorily presented items more than does an auditorily interfering saying task. This greater visual interference is obtained when (1) the stimuli provide up-down contour information, and (2) the visual task requires the subject to recall according to a different and incongruous contour. It did not matter whether the presented item was a melody (Experiment 1) or a spoken contour consisting only of the words "up" and "down" (Experiment 2). Yet, recall of spoken sentences containing no up-down information was not interfered with visually. These results show that melody recall is disrupted by visual pointing only inasmuch as this task interfered with contour, which is expressed by melodies but is not unique to it.

In Experiment 3, in which stimuli and tasks were controlled regarding degree of interfreence with contour, there appeared to be evidence-although it was not decisive-for an auditory interference effect occurring with melodic items. Although the more cognitively complex saying task was always slower than the pointing one, this difference was considerably larger for melodies than for visual contours. This result is consistent with the hypothesis that melodies are stored auditorily in shortterm memory, and that the saying task produces auditory interference with the recall of melodies but not of visual contours. Several problems with the auditory interference notion were discussed under Experiment 3. These problems, it was argued, applied less to the simpler congruous contour condition than to the incongruous one, which required the subjects to perform an extra inversion transformation during recall. The most serious problem was the interacting-difficulty-levels explanation, which could not be entirely ruled out even for congruous contours.

The importance of considering contour-related interference apart from that related to modality cannot be stressed enough. When contour condition was made a separate factor in Experiment 3, it was shown that when either the pointing or saying task required a contour incongruous with either visual or melodic stimuli, recall was interfered with. This effect was highly significant for both response time and accuracy data.

Indeed, contour interference appears to be far more convincing in explaining the results of all three experiments than does interference specific to either the visual or the auditory modality. In the first two experiments, the visual interference task slowed down recall of both melodies and spoken contours (as compared with sentence recall), implying that it functioned to disrupt memory for contour. In the last experiment, the main effect of having the subjects invert contour clearly made recall more difficult. This result also implies a general contour interference. Only when contour interference was eliminated, in the congruent condition of Experiment 3, was there evidence consistent with a specifically auditory interference. Even this evidence, as discussed earlier, was not entirely conclusive.

Two unanticipated effects obtained in Experiment 3 are 
worth special comment. The first of these is that visual contours were recalled both more quickly and more accurately than the same up-down patterns presented as melodies, regardless of the type of recall task. This effect contrasts with the results of many verbal memory studies, in which auditory presentation of stimuli such as words or letters leads to more accurate memory than does visual presentation of the same stimuli. This auditory primacy for verbal materials, known as the modality effect, has been well-established with several short-term memory techniques, including free recall (Gathercole, Gregg, \& Gardiner, 1983; Murdock \& Walker, 1969), serial recall (Cornballis, 1966; Watkins \& Watkins, 1980), and recognition (Kirsner \& Craik, 1971; Murdock 1968). In Experiment 3, in which recall of up-down contour was tested, the reverse effect was obtained. This finding merits further research. Although the visual contours and melodies had the same up-down patterns, there may have been other factors contributing to the effect. For instance, the up-down changes in the melodies might not have been as discriminable as those in the visual contours. Increasing pitch intervals could possibly lead to improved recall of the melodies. Assuming, however, that the superior recall of the visual patterns is due to visiospatial, as opposed to auditory, presentation, this visual primacy effect for contour would be interesting because it would represent a reversal of the traditional modality effect obtained with verbal materials.

Another unexpected result of Experiment 3 is the materials $\times$ contour condition interaction. Visual contours are more quickly inverted than are melodies, regardless of recall task. That is, the difference between the faster congruous reports of contour and the slower incongruous ones is less for visual items than for melodies. Dowling $(1972,1978)$, in discussing the ability of subjects to recognize melodic inversions and other transformations, speculated that they might use a visual imagery process similar to the "mental rotations" shown by Shepard and Metzler (1971). The present results indicated instead that inversions are definitely easier to perform with visual items, even though Dowling's subjects may well have been attempting visual imagery in recognizing melody manipulations.

Both of the effects just discussed-the main-effect recall superiority of visual over auditory contours, and the faster inversion of visual iems-suggest that the efficiency of contour recall may well depend on the form of stimulus input, even in the absence of strong evidence for modalityspecific memory coding. The visual displays recalled by the subjects in Experiment 3 were apparently more conducive to the abstraction of contour information than were the melodies, regardless of how that information might have been represented in memory storage. Currently, this author and his colleagues are beginning to use the shortterm recognition technique for the further study of contour memory. Recognition, which avoids the problem of unequal output characteristics of different recall tasks, should be a good method for investigating the effects of both input modality and type of interference.

\section{REFERENCES}

Bartlett, J. D., \& Dowling, W. J. (1980). The recognition of transposed melodies: A key-distance effect in developmental perspective. Journal of Experimental Psychology: Human Perception and Performance, 6, 501-515.

BrooKs, L. R. (1968). Spatial and verbal components of the act of recall. Canadian Journal of Psychology, 22, 349-368.

ConRad, R. (1964). Acoustic confusion in immediate memory. British Journal of Psychology, 55, 75-84.

Cornballis, M. C. (1966). Rehearsal and decay in immediate recall of visually and orally presented items. Canadian Journal of Psychology, 20, 43-51.

DAVIES, J. B., \& JENNIN Gs, J. (1977). Reproduction of familiar melodies and the perception of tonal sequences. Journal of the Acoustical Society of America, 61, 534-541.

Deutsch, D. (1970). Tone and numbers: Specificity of interference in immediate memory. Science, 168, 1604-1605.

Dowling, W. J. (1972). Recognition of melodic transformations. Perception \& Psychophysics, 12, 417-421.

Dowling, W. J. (1978). Scale and contour: Two components of a theory of melodies. Psychological Review, 85, 341-354.

Dowling, W. J., \& BARTLETT, J. C. (1981). The importance of interval information in long-term memory for melodies. Psychomusicology, 1, 30-49.

Gathercole, S. E., GregG, V. H., \& Gardiner, J. M. (1983). Influences of delayed distraction on the modality effect in free recall. British Journal of Psychology, 74, 223-232.

HAIR, H. I. (1977). Discrimination of tonal direction on verbal and nonverbal tasks by first grade children. Journal of Research in Music Education, 25, 197-210.

KirsNer, K., \& CraiK, F. (1971). Naming and decision processes in short-term recognition memory. Journal of Experimental Psychology, 88, 149-157.

LAughery, K. R., Welte, J. R., \& SPEctor, A. (1973). Acoustic and visual coding in primary and secondary memory. Journal of Experimental Psychology, 99, 323-329.

Massaro, D. W. (1970). Consolidation and interference in the perceptual memory system. Perception \& Psychophysics, 7, 153-156.

MURDOCK, B. B. (1968). Modality effects in short-term memory: Storage or retrieval. Journal of Experimental Psychology, 77, 79-86.

MuRDock, B. B., \& WalKer, K. O. (1969). Modality effects in free recall. Journal of Verbal Learning and Verbal Behavior, 8, 665-676.

OHLSSON, K., \& RonNBerg, J. (1981). Processing allocation of modality specific information. Umea Psychological Reports, No. 159, 1-16.

ShePARD, R. N., \& MetZler, J. (1971). Mental rotation of threedimensional objects. Science, 169, 701-703.

Watkins, O. C., \& WatkINS, M. J. (1980). The modality effect and echoic persistence. Joumal of Experimental Psychology: General, 109, 251-278.

WilliaMs, D. B. (1982). Auditory cognition: A study of similarities in memory processes for musical tones and spoken words. Bulletin of the Council for Research in Music Education, 71, 30-44.

\section{NOTES}

1. Frequencies (in hertz) are: $F_{4}=349, G_{4}=392, A_{4}=440, B_{4}=$ $494, C_{5}=524, D_{5}=587, E_{5}=660, F_{5}=698, G_{5}=784, A_{3}=$ 880 , and $B_{5}=988$.

2. Two assistants served as independent recorders. Judges agreed on over $99 \%$ of the responses. Responses were scored only for correctness of direction. For each subject, the chance probability was $50 \%$ correct responses.

(Manuscript received November 1, 1983; revision accepted for publication April 3, 1984.) 